\title{
Molecular Species of Phosphatidylglycerol from Leaves of Various Citrus Species
}

\author{
Jiro Sekiya, Katumasa NAGAi and Noboru Shimose \\ Department of Agricultural Sciences, Faculty of Agriculture, \\ Okayama University, Tsushima, Okayama 700, Japan \\ Received January 16, 1990
}

\begin{abstract}
Phosphatidylglycerol (PG) is a phospholipid characteristic of chloroplasts of higher plants. Molecular species of PG isolated from leaves of 7 Citrus species were examined and grouped into 5 classes. More than $90 \%$ of the total molecules were of the prokaryotic type synthesized in chloroplasts. Fatty acid compositions $(s n-1 / s n-2)$ of the major molecular species were $18: 1 / 16: 1(26-47 \%$ of the total molecules), 18:1/16:0 (11-35\%), and 16:0/16:1 (10-22\%), which were all prokaryotic. Molecular species with $18: 1$ at $s n-1$ were characteristic of Citrus leaf PG. Unsaturated prokaryotic molecular species of $(18: 1,18: 2$, and $18: 3) /(16: 0$ and $16: 1)$ accounted for $65-82 \%$, and saturated ones of $(16: 0$ and $18: 0) /(16: 0$ and $16: 1)$ for $19-32 \%$.
\end{abstract}

Phospholipids are one of the major lipid classes found in membranes of higher plant leaves. Recent development of analytical methods for lipids enabled us to identify molecular species of phospholipids readily, ${ }^{1-3}$ ) which suggests that molecular species composition is more important than fatty acid composition of individual lipid classes. It is rather difficult to estimate molecular species compositions from fatty acid compositions. 4 ,5) Some particular molecular species of $\mathrm{PG}$ are important in establishing the thermal phase transition temperature in herbaceous plant leaves. ${ }^{6,7)}$ Therefore, molecular species analyses of lipids are required for further understanding of relationships between chemical structure and function of membrane systems.

The genus Citrus contains some of the most important fruit trees cultivated throughout the world. Most Citrus species are evergreen woody plants and further improvements in photosynthetic activity and chilling resistance leading to higher yields and better quality of the fruits are desired. $\mathrm{PG}$ is a phospholipid characteristic of chloroplasts in leaves ${ }^{8)}$ and attention has been paid to this phospholipid in connection with photosynthetic activity ${ }^{9)}$ and chilling sensitivity. ${ }^{6)}$ However, little is known about molecular species of phospholipids in evergreen woody plant leaves such as Citrus species in spite of its importance. ${ }^{10-12)}$ This paper describes the molecular species composition of PG isolated from leaves of six Citrus species.

\section{Materials and Methods}

Plant materials. Leaves of Citrus junos cv. Tanenashimukaku yuzu were harvested at the Agricultural Experimental Station, Okayama University, in August; leaves of $C$. grandis (Tosa-buntan) in a greenhouse of our university in March; C. unshiu (unshu orange) on a local commercial farm in August; leaves of $C$. sinensis (Trovita orange) in the Akitsu Branch of the Fruit Tree Research Station, Ministry of Agriculture, Forestry and Fisheries, Japan in August; leaves of $C$. paradisi (grapefruit) and $C$. sinesis (navel orange) in a greenhouse of the ARCO Plant Cell Research Institute in Dublin, California, in November; C. paradisi (Pomelo) in the Botanical Garden of University of the Philippines at Los Baños, Philippines, in

Abbreviations: CL, cardiolipin; DGDG, digalactosyldiacylglycerol; MGDG, monogalactosyldiacylglycerol; PA, phosphatidic acid; PC, phosphatidylcholine; PE, phosphatidylethanolamine; PI, phosphatidylinositol; PG, phosphatidylglycerol; PS, phosphatidylserine; SQDG, sulfoquinovosyldiacylglyceride; 16:0, palmitic acid; 16:1, (E)-3-hexadecenoic acid; $17: 0$, margaric acid; $18: 0$, stearic acid; $18: 1$, oleic acid; $18: 2$, linoleic acid; $18: 3$, linolenic acid. 
January; leaves of C. amblycarpa (Djeroek limoe) on a commercial citrus farm in Bandung, Indonesia, in August.

Chemicals. Naja naja venom phospholipase $\mathrm{A}_{2}$, Bacillus cereus phospholipase $\mathrm{C}$, phospholipids (PC, PE, PI, PS, $\mathrm{CL}$, and $\mathrm{PA})$ and $\mathrm{PC}(18: 2 / 18: 2,16: 0 / 18: 2,18: 1 / 18: 1$, $16: 0 / 18: 1,18: 0 / 18: 1,18: 1 / 18: 0,18: 1 / 16: 0,18: 0 / 18: 0$, and $16: 0 / 16: 0$ for $s n-1 / s n-2)$ were obtained from the Sigma Chemical Co. (St. Louis, MO, U.S.A.), and PC (18:3/18:3, $18: 0 / 16: 0$, and 16:0/18:0) from Avanti Polar-Lipids, Inc. (Pelham, AL, U.S.A.). PC (18:0/16:1) was synthesized chemically from lyso-PC $(s n-1,18: 0)$ and 16:1. 3,5Dinitrobenzoylchloride was purchased from Wako Chemical Industries, Ltd., Tokyo. Precoated silica gel TLC plates were products of E. Merk (Silica gel 60) and Whatman Inc. (PLK5), and DEAE-Toyopearl 650M (Fractogel DEAE-650M) from Tosoh Ltd., Tokyo. All organic solvents used were glass-distilled.

Extraction and separation of lipids. Lipids were extracted from Citrus leaves essentially by the method of Allen and Good. ${ }^{13)}$ Total lipids obtained were separated into individual lipid classes by two-dimensional TLC on a Silica gel 60 plate and the fatty acid composition of each lipid was identified. PG was purified by preparative TLC on a PLK5 plate ${ }^{5)}$ or by column chromatography with DEAE-Toyopearl $650 \mathrm{M}$ coupled with the elution system described previously. ${ }^{14)}$ Each lipid class was confirmed by comparison of the $R f$ value with those of authentic specimens and spinach leaf lipids, and coloring reagents such as Dittmer's reagent for phosphorus groups, anthrone reagent for sugar residues, Dragendorf reagent for choline residues, and ninhydrin reagent for amino groups. ${ }^{5,15)}$

Fatty acid analysis. Fatty acid constituents of each lipid sample were transesterified with $5 \% \mathrm{H}_{2} \mathrm{SO}_{4}$ in $\mathrm{MeOH}$ for $2 \mathrm{hr}$ at $70^{\circ} \mathrm{C}$ and then analyzed with GLC by the method described previously. ${ }^{15}$,
Analysis of positional distribution of fatty acids. After treatment of PG with phospholipase $\mathrm{A}_{2}$, lysophosphatidylglycerol and free fatty acids were separated by TLC and then the fatty acid composition of each position was analyzed by the method described previously. ${ }^{15}$

Preparation of 3,5-dinitrobenzoyl derivatives of $P G$. Purified PG or authentic PC specimens were first hydrolyzed by phospholipase $\mathrm{C}$ and the resulting 1,2-diacylglycerides were esterified with 3,5-dinitrobenzoylchloride in anhydrous pyridine as described previously. ${ }^{1,153}$

HPLC analysis of 3,5-dinitrobenzoyl derivatives. 3,5Dinitrobenzoyl derivatives of $\mathrm{PG}$ were dissolved in small amounts of the mobile phase and injected into an HPLC mounting Nucleosil $3 \mathrm{C} 18$ column $(4.6 \times 150 \mathrm{~mm})$. Elution was achieved isocratically with acetonitrile-2-propanol $(80: 20, v / v)$. Each peak was collected and the fatty acid composition was analyzed by the method described above. Retention times of the peaks were compared with those of 3,5-dinitrobenzoyl derivatives of authentic specimens, phospholipids from pollen grains of trumpet lily (Lilium longiflorum $)^{5)}$ and tea, ${ }^{15)}$ and $\mathrm{PG}$ from tea leaves. ${ }^{4)}$

\section{Results and Discussion}

\section{Lipid composition}

Citrus leaves contained $20-30 \mathrm{mg}$ of extractable lipids per $1 \mathrm{~g}$ fresh weight. Major glycerolipids found in Citrus leaves were MGDG, DGDG, SQDG, PI, PG, PC, and PE, when a two-dimensionally developed TLC plate was stained with $\mathrm{I}_{2}$ vapor. PS, PA, and CL were also detected as minor ones. A typical glycerolipid composition of C. junos was shown in Table I. Seven glycerolipids listed in

Table I. Contents and Fatty Acid Compositions of Major Glycerolipids Isolated from C. junos Leaf

\begin{tabular}{|c|c|c|c|c|c|c|c|c|}
\hline \multirow{2}{*}{ Lipid class } & \multirow{2}{*}{$\begin{array}{c}\text { Content }^{a} \\
(\mu \mathrm{mol} / \mathrm{g} \text { fresh } w \mathrm{t})\end{array}$} & \multicolumn{7}{|c|}{ Fatty acid composition (mol\%) } \\
\hline & & $16: 0$ & $16: 1$ & $18: 0$ & $18: 1$ & $18: 2$ & $18: 3$ & Others \\
\hline MGDG & $1.73^{b}(37)^{c}$ & 8 & 0 & 3 & 3 & 5 & 80 & 1 \\
\hline DGDG & $1.10(23)$ & 36 & 0 & 16 & 4 & 3 & 39 & 2 \\
\hline SQDG & $0.61(13)$ & 49 & 0 & 15 & 10 & 4 & 20 & 2 \\
\hline $\mathrm{PG}$ & $0.37(8)$ & 29 & 27 & 10 & 29 & 2 & 2 & 1 \\
\hline $\mathrm{PC}$ & $0.26(6)$ & 47 & 1 & 14 & 20 & 8 & 8 & 2 \\
\hline $\mathrm{PE}$ & $0.17(4)$ & 37 & 0 & 13 & 16 & 17 & 13 & 4 \\
\hline PI & $0.44(9)$ & 56 & 0 & 6 & 9 & 10 & 16 & 3 \\
\hline
\end{tabular}

a Total lipids extracted were $21.3 \mathrm{mg} / \mathrm{g}$ fresh weight.

${ }^{b}$ Calculated from fatty acid composition by using 17:0 as an internal standard.

c Values in parentheses indicate \% distribution within the lipids listed. 
Table II. Fatty Acid Compositions of PG Isolated from Leaves of Various Citrus Species

\begin{tabular}{|c|c|c|c|c|c|c|c|}
\hline \multirow{2}{*}{ Species } & \multicolumn{7}{|c|}{ Fatty acid composition ( $\mathrm{mol} \%$ ) } \\
\hline & $16: 0$ & $16: 1$ & $18: 0$ & $18: 1$ & $18: 2$ & $18: 3$ & Others \\
\hline \multicolumn{8}{|l|}{ Arachicitrus TANAKA } \\
\hline C. grandis (Tosa-buntan) & 26 & 36 & 2 & 18 & 10 & 8 & $\operatorname{tr}^{a}$ \\
\hline C. paradisi (Grapefruit) & 34 & 20 & 4 & 30 & 7 & 3 & 2 \\
\hline C. paradisi (Pomelo) & 27 & 26 & 6 & 35 & 3 & $\operatorname{tr}$ & 3 \\
\hline C. sinensis (Navel orange) & 27 & 30 & 4 & 30 & 3 & 1 & 5 \\
\hline C. sinensis (Trovita orange) & 29 & 31 & 4 & 34 & 1 & 1 & 0 \\
\hline \multicolumn{8}{|l|}{ Metacitrus TANAKa } \\
\hline C. junos (Yuzu) & 32 & 32 & 8 & 24 & $\operatorname{tr}$. & 2 & 2 \\
\hline C. unshiu (Unshu) & 26 & 29 & 4 & 33 & 4 & 4 & 0 \\
\hline C. amblycarpa (Djeroek limoe) & 39 & 29 & 5 & 19 & 5 & 3 & 0 \\
\hline
\end{tabular}

a Detectable but less than $0.5 \%$.

Table I accounted for about $20 \%$ of total lipids extracted. Fatty acid compositions of the seven glycerolipids were analyzed after the individual lipid classes were separated by two-dimensional TLC. Major fatty acid constituents were 18:3 for MGDG; 16:0, 18:0, and 18:3 for DGDG and SQDG; $16: 0,16: 1$, and $18: 1$ for $P G$; $16: 0,18: 0$, and $18: 1$ for $P C ; 16: 0,18: 0,18: 1$, $18: 2$, and $18: 3$ for PE; and $16: 0,18: 1,18: 2$, and $18: 3$ for PI. Significant amounts of fatty acids with carbon numbers of 14,20 and more were not found in these glycerolipids.

\section{Leaf $P G$ in various Citrus species}

Fatty acid compositions of leaf PG were further examined in various Citrus species. Leaf PG occurred mostly in the chloroplast as well as MGDG, DGDG, and SQDG. ${ }^{8)} \mathrm{PG}$ was isolated from leaves by DEAE-Toyopearl $650 \mathrm{M}$ column chromatography followed by silica gel column chromatography. PG was one of the major phospholipids in all Citrus species examined. The fatty acid compositions of leaf PG obtained were similar among the various species examined (Table II). A large amount of 18: 1 was present in PG isolated from Citrus leaves. This agreed with earlier work done with Citrus species, ${ }^{10)}$ tea, ${ }^{4)}$ and some woody plants, ${ }^{16)}$ although 18:1 accounted for less than $10 \%$ of the total fatty acid constituents
Table III. Positional Distribution of Fatty ACids IN PG IsOlated FRom $C$. junos LeAF

\begin{tabular}{crrrrrr}
\hline \multirow{2}{*}{ Position } & \multicolumn{5}{c}{ Fatty acid composition (mol\%) } \\
\cline { 2 - 7 } & $16: 0$ & $16: 1$ & $18: 0$ & $18: 1$ & $18: 2$ & $18: 3$ \\
\hline$s n-1$ & 29 & 2 & 16 & 50 & 1 & 1 \\
$s n-2$ & 20 & 63 & 5 & 5 & 2 & 2 \\
\hline
\end{tabular}

in most herbaceous plants. ${ }^{12,14,17,18)}$ Three fatty acids $(16: 0,16: 1$, and $18: 1)$ accounted for $80-90 \%$ of the total constituents in 8 kinds of Citrus leaves (Table II). The fatty acid constituents $16: 0,16: 1,{ }^{* 1}$ and $18: 0$ were 58 $64 \%$ of the total fatty acids and polyunsaturated $18: 2$ and $18: 3$ were minor ones, probably due to use of leaves grown under warm condition.

$16: 1$ was exclusively located in the $s n-2$ position of the glycerol moiety and $18: 1$ in the $s n-1$ position (Table III). These results agreed with earlier work. ${ }^{4,12,19)}$ There was no apparent difference in fatty acid compositions between the two classes of Citrus species, arachicitrus and metacitrus (Table II).

\section{Molecular species of $P G$}

Isolated $\mathrm{PG}$ was converted to 3,5-dinitrobenzoyl derivatives and analyzed by HPLC. A typical elution profile of the derivatives

*1 Fatty acid $(16: 1)$ with an $(E)$-3-double bond was classified in the same group as $16: 0$ because $16: 1$ is closely related to $16: 1$ with respect to biosynthesis ${ }^{19)}$ and thermal phase transition ${ }^{20)}$ in $P G$. 
prepared from $C$. junos leaf $\mathrm{PG}$ is shown in Fig. 1. Each peak was collected and the fatty acid composition was analyzed by GLC after transmethylation. Retention times of the peaks were also compared with those of 3,5dinitrobenzoyl derivatives with known fatty acid compositions. We also assumed that the

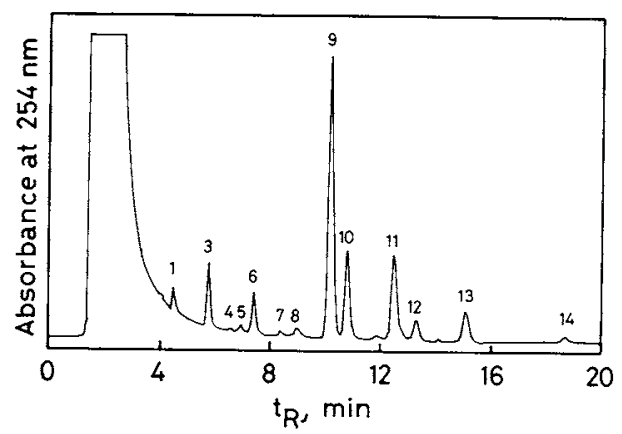

Fig. 1. HPLC Profile of 3,5-Dinitrobenzoyl Derivatives of PG Isolated from Leaves of C. junos Grown in Summer. Peak numbers are corresponding to Tables IV and $\mathrm{V}$. majority of $18: 1$ was located at $s n-1$ of the glycerol moiety and $16: 1$ at $s n-2$. The evidence for identification of each peak is summarized in Table IV.

Molecular species compositions of $\mathrm{PG}$ are shown in Table V. PG molecules isolated from leaves of the various Citrus species were grouped in 5 classes biosynthetically based on fatty acid composition $(s n-1 / s n-2) ; 18: \mathrm{m} / 18: \mathrm{n}$ ( $<4 \%$ of the total molecules), $18: \mathrm{m} /(16: 0$ and $16: 1)(65-82 \%), 18: 0 /(16: 0$ and $16: 1)(3-$ $7 \%), 16: 0 /(16: 0$ and $16: 1)(1428 \%)$, and $16: 0 / 18: \mathrm{m}(<9 \%)$, where the numbers of $\mathrm{m}$ and $\mathrm{n}$ are 1,2 , and 3 . Among them, $18: \mathrm{m} /(16: 0$ and $16: 1), 18: 0 /(16: 0$ and $16: 1)$, and $16: 0 /(16: 0$ and $16: 1)$, which are so-called prokaryotic molecules, ${ }^{21)}$ are synthesized in the chloroplast. These 3 classes of PG molecules accounted for more than $90 \%$ of the total molecules in all species examined (Table V). Thus, $\mathrm{PG}$ isolated from the leaves reflects

Table IV. Identification of HPlC Peaks of 3,5-Dinitrobenzoyl Derivatives of PG

\begin{tabular}{|c|c|c|c|c|c|c|c|c|c|}
\hline \multirow{2}{*}{$\begin{array}{c}\text { Peak }^{a} \\
\text { no }\end{array}$} & \multirow{2}{*}{$\begin{array}{l}\text { Relative } \\
\text { retention } \\
\text { time }\end{array}$} & \multirow{2}{*}{ Evidence $^{b}$} & \multicolumn{6}{|c|}{ Fatty acid composition (mol\%) } & \multirow{2}{*}{$\begin{array}{c}\text { Fatty acid } \\
s n-1 / s n-2\end{array}$} \\
\hline & & & $16: 0$ & $16: 1$ & $18: 0$ & $18: 1$ & $18: 2$ & $18: 3$ & \\
\hline 1 & 0.33 & A, B & n.d..$^{c}$ & & & & & & $18: 3 / 18: 3$ \\
\hline 2 & 0.40 & B & n.d. & & & & & & $(18: 2 / 18: 3)^{d}$ \\
\hline 3 & 0.45 & $\mathrm{D}$ & 8 & 56 & 0 & 0 & 0 & 36 & $18: 3 / 16: 1$ \\
\hline 4 & 0.49 & A, B & n.d. & & & & & & $18: 2 / 18: 2$ \\
\hline 5 & 0.52 & $\mathrm{~B}, \mathrm{D}$ & 32 & 9 & 4 & 4 & 0 & 56 & $18: 3 / 16: 0^{e}$ \\
\hline 6 & 0.54 & $\mathrm{D}$ & 5 & 36 & 0 & 7 & 50 & 2 & $18: 2 / 16: 1$ \\
\hline 7 & 0.57 & n.d. & n.d. & & & & & & \\
\hline $8^{f}$ & 0.69 & $\begin{array}{l}\text { A, B, D } \\
\text { D }\end{array}$ & 31 & 5 & 15 & 0 & 36 & 13 & $\begin{array}{r}18: 2 / 16: 0^{g} \\
(18: 3 / 18: 0)\end{array}$ \\
\hline 9 & 0.77 & C, D & 5 & 41 & 1 & 47 & 5 & 1 & $18: 1 / 16: 1$ \\
\hline 10 & 0.82 & $\mathrm{D}$ & 51 & 35 & 3 & 6 & 4 & 1 & $16: 0 / 16: 1$ \\
\hline 11 & 0.94 & $A, D$ & 47 & 1 & 2 & 47 & 2 & 1 & $18: 1 / 16: 0$ \\
\hline 12 & 1.00 & A, D & 85 & 0 & 0 & 8 & 7 & 0 & $16: 0 / 16: 0$ \\
\hline 13 & 1.05 & $A, D$ & 8 & 42 & 38 & 0 & 12 & 0 & $18: 0 / 16: 1$ \\
\hline 14 & 1.38 & A & n.d. & & & & & & $(18: 0 / 16: 0)^{h}$ \\
\hline
\end{tabular}

a Peak numbers are the same as those in Fig. 1.

$b$ A, comparison of the retention time with those of authentic specimens; $\mathrm{B}$, comparison of the retention time with those of pollen lipids of lily ${ }^{5}$ and/or tea ${ }^{15} ; \mathrm{C}$, comparison of the retention time with those of the tea leaf PG derivatives ${ }^{4)}$; , fatty acid analysis.

c Not determined.

d Mixture of $18: 2 / 18: 3$ and $18: 3 / 18: 2$.

e A possibility of the presence of $16: 0 / 18: 3$ could not be excluded.

$f$ Mixture of $18: 2 / 16: 0,18: 3 / 18: 0$ and $18: 0 / 18: 3$.

${ }^{g}$ A possibility of the presence of $16: 0 / 18: 2$ could not be excluded.

${ }^{h}$ Mixture of $18: 0 / 16: 0$ and $16: 0 / 18: 0$. 
Table V. Molecular Species Compositions of PG from Various Citrus Leaves

\begin{tabular}{|c|c|c|c|c|c|c|c|c|}
\hline \multirow{2}{*}{$\begin{array}{c}\text { Peak } \\
\text { no }\end{array}$} & \multirow{2}{*}{$\frac{\text { Fatty acid }}{\text { C-1 C-2 }}$} & \multicolumn{7}{|c|}{ Molecular species composition (mol\%) } \\
\hline & & C. grandis & $\begin{array}{l}\text { C. paradisi } \\
\text { (Pomelo) }\end{array}$ & $\begin{array}{l}\text { C. sinensis } \\
\text { (Navel) }\end{array}$ & $\begin{array}{l}\text { C. sinensis } \\
\text { (Trovita) }\end{array}$ & C. junos & C. unshiu & $\begin{array}{c}C . \\
\text { amblycarpa }\end{array}$ \\
\hline 1 & $18: 318: 3$ & $\operatorname{tr}^{a}$ & 2 & 1 & 1 & 2 & tr. & 2 \\
\hline 2 & $(18: 2 / 18: 3)^{b}$ & 2 & 0 & 2 & tr. & 0 & tr. & 0 \\
\hline 3 & $18: 3 \quad 16: 1$ & 10 & 4 & 3 & 4 & 6 & 3 & 7 \\
\hline 4 & $18: 218: 2$ & 1 & 0 & 1 & 0 & tr. & tr. & 0 \\
\hline 5 & $18: 316: 0$ & 3 & 2 & 2 & 1 & 1 & 2 & 3 \\
\hline 6 & $18: 216: 1$ & 9 & 4 & 5 & 5 & 5 & 2 & 5 \\
\hline 7 & nd & tr. & 0 & 0 & tr. & tr. & tr. & tr. \\
\hline 8 & $\begin{array}{c}18: 216: 0^{b} \\
(18: 3 / 18: 0)^{b}\end{array}$ & 6 & 2 & 3 & 1 & 1 & 3 & 1 \\
\hline 9 & $18: 1 \quad 16: 1$ & 26 & 32 & 36 & 47 & 43 & 29 & 46 \\
\hline 10 & $16: 016: 1$ & 22 & 11 & 11 & 11 & 14 & 10 & 10 \\
\hline 11 & $18: 1 \quad 16: 0$ & 11 & 34 & 23 & 21 & 17 & 35 & 20 \\
\hline 12 & $16: 016: 0$ & 6 & 7 & 9 & 4 & 4 & 9 & 4 \\
\hline 13 & $18: 016: 1$ & 3 & 2 & 3 & 4 & 6 & 4 & 4 \\
\hline 14 & $18: 016: 0$ & 1 & 1 & 1 & 1 & 1 & 3 & 1 \\
\hline
\end{tabular}

a Detectable but less than $0.5 \%$.

${ }^{b}$ See footnotes in Table IV.

chloroplast PG.

Major molecular species of $\mathrm{PG}$ common in all species examined were $18: 1 / 16: 1(26-$ $47 \%), 18: 1 / 16: 0(11-35 \%)$, and $16: 0 / 16: 1$ $(10-22 \%)$. In addition $C$. grandis contained $18: 3 / 16: 1(10 \%)$ and $18: 2 / 16: 1(9 \%)$, and $C$. sinensis (navel) and C. unshiu 16:0/16:0 (9\%). These major molecular species were all prokaryotic. Occurrence of $18: 1 /(16: 0$ and $16: 1)$ in large amounts $(37-68 \%)$ is characteristic of Citrus leaf PG as well as leaf PG of evergreen woody plants, tea $(40-70 \%),{ }^{4)}$ and avocado $(46 \%) .{ }^{12)}$ The sum of prokaryotic unsaturated molecular species, $18: 1 /(16: 0$ and $16: 1)$ and $(18: 2$ and $18: 3) /(16: 0$ and $16: 1)$, accounted for $65-82 \%$ of the total molecules, while saturated species, $16: 0 /(16: 0$ and $16: 1)$ and $18: 0 /(16: 0$ and $16: 1$ ) were $19-32 \%$ (Table V). Thus, the ratios of unsaturated/saturated molecular species of Citrus leaf PG presented here are similar to those of chilling-resistant herbaceous plants such as pea and lettuce rather than chilling-sensitive herbaceous plants. ${ }^{6)}$

In Table II, relative contents of saturated fatty acid constituents range from 58 to $64 \%$
(Table II), although those of saturated molecular species were $19-32 \%$. This indicates that estimation of molecular species composition from whole fatty acid composition is very difficult. Therefore, fatty acid compositions of glycerolipids in some earlier works should be re-examined in terms of molecular species compositions. Further investigation of the relationship between response to environmental stress and molecular species composition is now in progress.

Acknowledgments. We express our thanks to Dr. W. Padolina, Mrs. E. C. Bugante, and E. C. Marfori of Biotech at the University of the Philippines at Los Baños, Philippines, for preparing C. paradisi leaf lipids; to Dr. E. Noerhadi, Mr. D. Sasmitamihardja, and Mrs. A. H. Siregar of Institute Teknologi Bandung, Indonesia, for preparing $C$. amblycarpa leaf lipids; and to Dr. S. Kobayashi of the Akitsu Branch of the Fruit Tree Research Station, Ministry of Agriculture, Forestry, and Fisheries of Japan, for preparing $C$. sinensis leaf lipids. We also thank Dr. J. B. Mudd of the ARCO Plant Cell Research Institute, Dublin, California, USA, and Dr. N. Murata of the National Institute for Basic Biology, Okazaki, Japan, for their useful advice and discussions on lipid analysis. This work was supported in part by the cooperative research programs sponsored by the Japan Society 
for the Promotion of Science (JSPS) and also by a Grant-in-Aid for General Scientific Research (No. 62304004) from the Ministry of Education, Science and Culture of Japan.

\section{References}

1) M. Kito, H. Takamura, H. Narita and R. Urade, $J$. Biochem., 98, 327 (1985).

2) J. Kesselmeier and E. Heinz, Anal. Biochem., 144, 319 (1985).

3) L. A. Smith and G. A. Thompson, Jr., in "High Performance Liquid Chromatography in Plant Sciences," ed. by H. F. Linskens and J. F. Jackson, Springer-Verlag, Berlin, 1987, pp. 149-169.

4) J. Sekiya, H. Koiso, A. Morita and A. Hatanaka, in "The Metabolism, Structure, and Function of Plant Lipids," ed. by P. K. Stumpf, J. B. Mudd and W. D. Nes, Plenum Press, New York, 1987, pp. $377-380$.

5) J. Sekiya, K. Yamashita and N. Shimose, Agric. Biol. Chem., 54, 191 (1990).

6) N. Murata, Plant Cell Physiol., 24, 81 (1983).

7) N. Murata and J. Yamaya, Plant Physiol., 74, 1016 (1984).

8) J. L. Harwood, in "The Biochemistry of Plants," Vol. 4, ed. by P. K. Stumpf, Academic Press, New York,
1980, pp. 1-55.

9) J. P. Dubacq and A. Trémolières, Physiol. Vég., 21, 293 (1983).

10) H. E. Nordby and G. Yelenosky, Phytochemistry, 23, 41 (1984).

11) H. E. Nordby and G. Yelenosky, Phytochemistry, 24, 1675 (1985).

12) P. G. Roughan, Plant Physiol., 77, 740 (1985).

13) C. F. Allen and P. Good, in "Methods in Enzymology," Vol. 23, ed. by A. San Pietro, Academic Press, New York, 1971, pp. 523-547.

14) N. Murata, N. Sato, N. Takahashi and Y. Hamazaki, Plant Cell Physiol., 23, 1071 (1982).

15) J. Sekiya, K. Yamashita, S. Nakagawa, Y. Shibata and A. Hatanaka, Agric. Biol, Chem., 52, 243 (I988).

16) Y. Tasaka, I. Nishida, S. Higashi, T. Beppu and N. Murata, Plant Cell Physiol., 31, 545 (1990).

17) J. R. Kenrick and D. G. Bishop, Plant Physiol., 81, 946 (1986).

18) J. R. Kenrick and D. G. Bishop, Phytochemistry, 25, 1293 (1986).

19) B. W. Nichols, P. Harris and A. T. James, Biochem. Biophys. Res. Commun. 21, 473 (1965).

20) D. G. Bishop and J. R. Kenrick, Phytochemistry, 26, 3065 (1987).

21) P. G. Roughan and C. R. Slack, Ann. Rev. Plant Physiol., 33, 97 (1982). 